\title{
Cytokines and their Role in Health and Disease: A Brief Overview
}

\begin{abstract}
Cytokines are small, non-structural proteins with low molecular weights which have a complex regulatory influence on inflammation and immunity. It has long been considered that development of immune and inflammatory response involves hematopoietic cells, lymphoid cell, and various pro-inflammatory and anti-inflammatory cells, and cytokines mediate the complex interactions of these cells. Cytokines are the intercellular messengers in the immune system where they integrate function of several cell types in various body compartments into a coherent immune response. They have evolved over the years and now include the interferons, the interleukins, the chemokine family, mesenchymal growth factors, the tumor necrosis factor family and adipokines. Earlier known as 'soluble factors', cytokines are produced by and elicit a response from every cell, with the lone exception of the red blood cell. In response to various stimulus cytokines are secreted from various cells including white blood cells. Pleiotropism is the hallmark of a cytokine and there are failures and successes of cytokines and related agents as therapeutic agents. In the membrane of target cells they have specific receptors for their signal transduction and regulatory functions. Beyond the innate and adaptive immunity, cytokines has a major role in many diverse functions including immune cell differentiation, inflammation, angiogenesis, tumorigenesis, neurobiology, viral pathogenesis etc. In addition to inflammation, immunity and infections, cytokines have now expanded their domain to atherosclerosis and cancer. Thus, cytokines may be useful biomarkers for health and disease and act as diagnostic, prognostic and therapeutic agents. This review will highlight the impact of cytokines in various physiological actions, pathophysiological states, therapeutics and the complex interactions between brain and immune cells.
\end{abstract}

Keywords: Cytokines; Cytokine receptors; IFN $\gamma$; Cytokines and disease; Cytokines in therapy; Cytokine toxicity; T cells; Tr1; TNF- $\alpha$

\author{
Review Article \\ Volume 4 Issue 2 - 2016 \\ Kavita Gulati, Sreemanti Guhathakurta, \\ Jagdish Joshi, Nishant Rai and Arunabha \\ Ray* \\ Department of Pharmacology, University of Delhi, India \\ *Corresponding author: Arunabha Ray, Director-Professor \\ \& Head, Department of Pharmacology, Vallabhbhai Patel \\ Chest Institute, Faculty of Medicine, University of Delhi, \\ Delhi-110 007, India; Tel: 91-9818037595; Fax: 91-11- \\ 27666549; Email: arunabha14@yahoo.co.in
}

Received: August 07, 2016 | Published: October 19, 2016
Abbreviations: Tr1: Treg Cells Type 1; IFN $\gamma$ : Interferon- $\gamma$; CTL: Cytotoxic T-Cells; MDM: Monocyte Derived Macrophages; VCAM-1: Vascular Cell Adhesion Molecule; AMPAR: AMPA-Type Glutamate Receptors; VEGF: Vascular Endothelial Growth Factor; CGD: Chronic Granulomatous Disease

\section{Introduction}

The story of cytokines goes back to last century when interferon was discovered first time. Cytokines are pleiotropic proteins or small glycoproteins with a molecular weight less than $30 \mathrm{kDa}(<200$ amino acids) Cytokines are produced by a number of cell types, such as leukocytes which regulate immunity, inflammation and hematopoiesis [1]. About 200 cytokines are recognized to date. They have a high degree of $\alpha$ helical structure and the molecules share a similar polypeptide fold with four $\alpha$ helical bundles. They are categorized on the basis from which they are produced either from Th1 cells or Th2 cells. Recently a third subset of Th cells (Th17) and T regulatory cells (Treg) are categorized which show different cytokine profile from Th1 and Th2 cells (Figure 1). It secretes IL-17, IL-17F, IL-22 and IL25 . Treg cells type 1 (Tr1) secrete mainly IL-10and IFN- $\gamma$, IL-5 in lesser amount and very low level of TGF- $\beta$ and IL-2. Tr3 subset of Treg (also called Th3 cells) produces preferentially TGF- $\beta$ and lesser amounts of IL-10 [2]. According to their secretion they are classified into lymphokines (cytokines that are secreted by T cells and regulate the immune response), proinflammatory cytokines (cytokines that amplify and perpetuate the inflammatory process), growth factors (cytokines that promote cell survival and result in structural changes in the airways), chemokines (cytokines that are chemotactic for inflammatory cells) and anti-inflammatory cytokines (cytokines that negatively modulate the inflammatory response) [3].

The history of cytokine development shows them as soluble factors produced by one cell and acting on another cell, and recognition of the activity of cytokines was established (19401960). It was soon apparent that the production of these factors could be regulated by activation with an antigen or a nonspecific mitogen. A standardized nomenclature designating the cytokines as interleukins in relation to their role among leukocytes was also developed, and the first to be named were IL-1 and IL-2. Leukemic monocyte lines were chosen for the development of interleukin-1 and T-cell lymphomas for the production of IL-2 [4]. Today, they are recognized as integral membrane proteins and some cytokines may never be released from cells. Advancing 
knowledge has highlighted the complexity and biphasic nature of cytokines - and the same molecule may have beneficial as well as detrimental effects. For example, interferon- $\gamma$ (IFN $\gamma$ ), which is essential for defense against several intracellular microorganism such as Mycobacterium tuberculosis, is also a major cytokine in the pathogenesis of several autoimmune diseases. IL-2 is needed for the generation of cytotoxic T-cells (CTL) and forms the basis for several vaccines but the same cytokine drives graft versus host disease and limits the success of bone marrow transplantation [5].

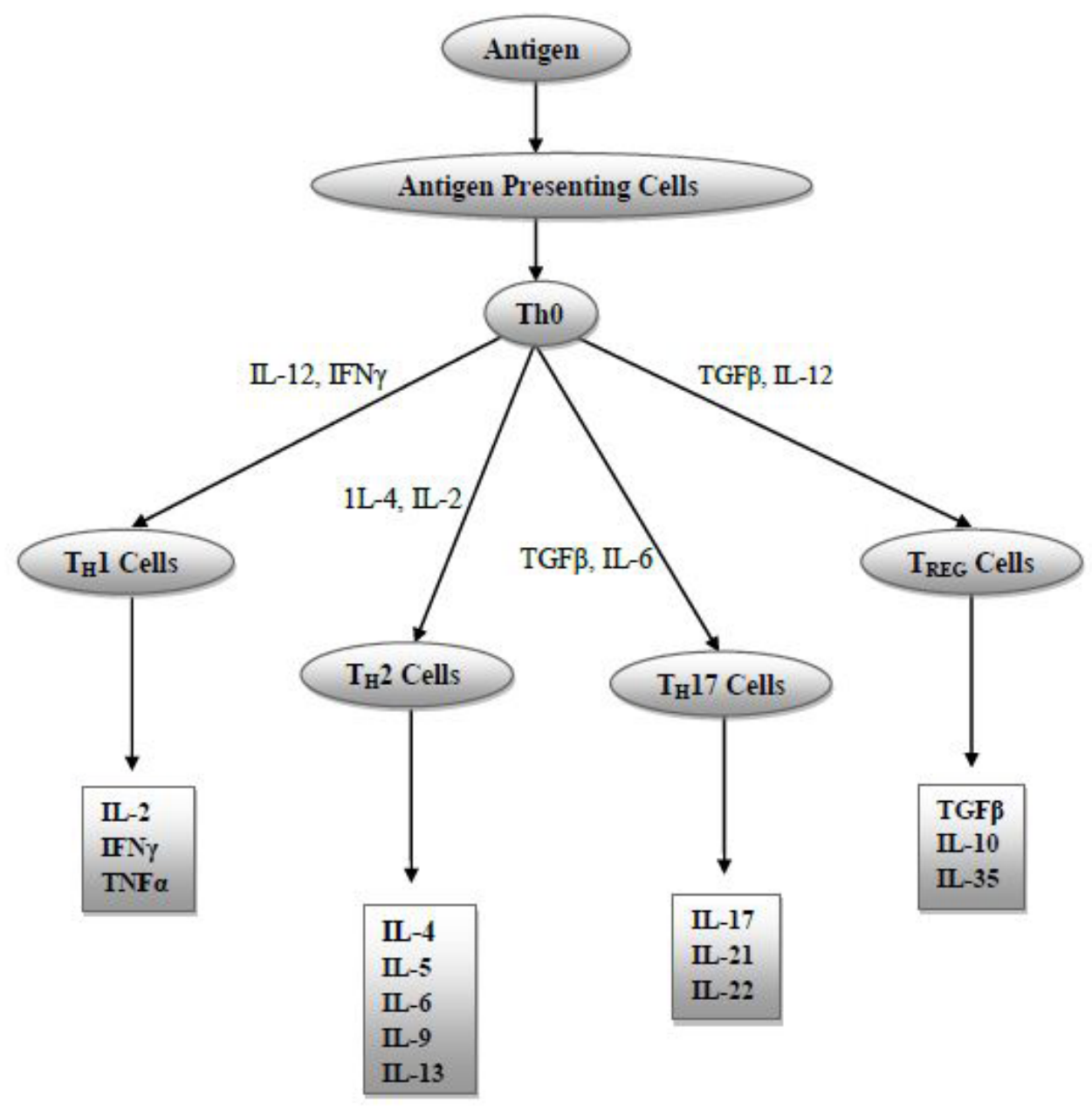

Figure 1: Differentiation of naive T-cells

\section{Cytokine Receptors}

The cytokines exert various biological effects through receptors present on the membranes of responsive target cells. These receptors have an extracellular domain, a cytoplasmic domain and a single membrane spanning domain. There is the presence of conserved amino acid sequence motifs on the extracellular domain and these motifs include four conserved cysteine residues. There is also the presence of two polypeptide chains. One is the cytokine specific $\alpha$ subunit and other is a signal transducing $\beta$ subunit.
The nature of the target cell to which the cytokines bind is determined by the presence of specific membrane receptors. Cytokines and their receptors exhibit very high affinity for each other and possess dissociation constants ranging from $10^{-10}$ to $10^{-12} \mathrm{M}$ and for this reason of high affinity, biological effects are produced by cytokines in picomolar concentrations. They exhibit autocrine, paracrine, and endocrine actions and mediate cellular intercommunication [6]. The intensity or duration of immune response is regulated by either stimulating or inhibiting the activation, proliferation and differentiation of various cells and thus regulating the secretion of antibodies or other cytokines. 
The physiological responses of cytokines on binding to receptors include development of cellular and humoral immune response, induction of inflammatory response, regulation of hematopoiesis, control of cellular proliferation and differentiation and induction of wound healing. Other than this, the cytokines often induce the synthesis of other cytokines resulting in a cascade of activity in which the latter cytokines influence the activity of the former cytokines which secreted it. Finally, they function for a very limited period of time due to their short half-life in the blood stream and extracellular fluids [4].

\section{Peripheral inflammatory response of cytokines}

Inflammation is an immunological defence mechanism of the body against injury, infection and allergy marked by immigration of WBCs and release of chemical toxins. Acute inflammatory response is characterized by rapid onset and is of short duration. This is accompanied by a systemic response known as the acute phase response which is characterized by alterations in the levels of several plasma proteins. Activation of tissue macrophages with the release of cytokines like TNF- $\alpha$, IL- 1 and IL- 6 occurs during this kind of a response. These three cytokines act on the fibroblasts and endothelial cells inducing coagulation and increase the vascular permeability of the cells. An increased expression of adhesion molecules occur on vascular endothelial cells by TNF- $\alpha$ and IL-1. IL-1 stimulates the expression of adhesion molecules, ICAM- 1 and VCAM-1 that binds to lymphocytes and monocytes. Circulating neutrophils, lymphocytes and monocytes recognize adhesion molecules and travel through the blood vessel walls to the tissue spaces. TNF- $\alpha$ and IL- 1 also acts on macrophages and endothelial cells inducing the expression of IL-8 which is responsible for the infiltration of neutrophils and is also a potent chemotactic factor. TNF- $\alpha$, IL-1 and IL- 6 act together on the hypothalamus in the brain inducing the fever response and the brain levels of these cytokines are also elevated. TNF- $\alpha$ also acts on vascular endothelial cells and macrophages inducing the expression of colony stimulating factors (M-CSF, G-CSF and GM-CSF) and finally leads to hematopoiesis resulting in an increased expression of necessary white blood cells to fight this kind of an infection. IFN- $\gamma$ and TNF- $\alpha$ are produced by Th1 cells due to prolonged persistence of an antigen and leads to chronic inflammatory reaction. Macrophages play a major role in the regulation of the chronic inflammatory response. Activation of macrophages by IFN- $\gamma$ induces increased expression of class II MHC molecules, increased cytokine production and increased microbicidal activity. Once the macrophages become activated they serve as effective antigen presenting cells and are more effective in killing the intracellular pathogens. TNF- $\alpha$ is the principal cytokine produced by activated macrophages and have direct cytotoxic effects on tumor cells. When the macrophages become activated by IFN- $\gamma$ they stimulate an increased transcription of TNF- $\alpha$ gene and increase the stability of TNF- $\alpha$ mRNA. Both these effects lead to an increased expression of TNF- $\alpha$ which synergistically acts with IFN- - thus initiating this kind of a response. As a result of this initiation, there is an increased expression of intercellular adhesion molecules like ICAM-1, ELAM-1 and class I MHC which results in the recruitment of large number of cells during this chronic inflammatory response [4].

\section{Role of cytokines in diseases}

Bronchial asthma: Bronchial asthma is an inflammatory disease of the airways and is associated with bronchial hyper reactivity and reversible airway obstruction. Studies indicate that $\mathrm{T}$ cell derived cytokine production rather than eosinophil influx or $\operatorname{IgE}$ synthesis is usually related to altered airway behavior [7]. There is an increase in the number of CD4+ Th cells in the airways which are predominantly of the Th2 subtype. Th2 cells are characterized by secretion of IL-4, IL-5, IL-9 and IL-13 [3]. Increased expression of pro-inflammatory cytokine TNF- $\alpha$, enhances the inflammatory process and has been linked to disease severity. IL-4 is the key cytokine in asthma and is involved in Th2 cell differentiation and IgE production. It stimulates the mucus producing cells and fibroblasts implicating its role in the pathogenesis of airway remodeling. In atopic asthmatics, IL-4 induces airway eosinophilia and causes bronchial hyper responsiveness. IL- 5 is the primary cytokine involved in the production, differentiation, maturation and activation of eosinophils. It is crucial for inducing eosinophil infiltration in the airways. IL-13 is present in increased amounts in asthmatic airways and has very similar biological activities to IL-4 [7]. IL-4 induces IgE dependent mast cell activation involved in immediate type allergic/hypersensitivity reactions. In the asthmatic lung, IL-4 promotes cellular inflammation by induction of vascular cell adhesion molecule (VCAM-1) on the vascular endothelium [8].

Human studies have also shown increased expression of IL-9 in the bronchial biopsy sample of asthmatics. In vitro data indicates that IL-9 stimulates proliferation of activated T cells, enhances production of IgE from B cells, and promotes proliferation and differentiation of mast cells. TNF- $\alpha$ causes leukocyte recruitment, through up-regulation of adhesion molecules and induction of cytokine and chemokine synthesis. Elevated levels of TNF- $\alpha$ have been detected in sputum and BAL fluid from asthmatics. It has the potential to stimulate fibroblasts or smooth muscle cells indicating its role in the pathogenesis of airway remodeling [7]. IL-10 is a pleiotropic cytokine which up-regulates the expression of anti-inflammatory cytokine IL-1ra and suppresses the proinflammatory functions of cytokines like TNF- $\alpha$, IL-1, IL- 6 and IL-8 [9]. So, this might have some kind of beneficial effect during airway remodeling as it has been shown to reduce collagen synthesis and vascular smooth muscle proliferation. IL-12 is the necessary cofactor for Th1 development. It primarily regulates Th1 cell differentiation while suppressing the expansion of Th2 cells. IFN- $\gamma$, a Th1 marker, exerts inhibitory effects on Th2 cell differentiation [7].

Pleiotropic activities of Th2 cytokines in allergic asthma: When a naive $\mathrm{T}$ cell encounters an antigen in presence of antigen presenting cells like macrophages, dendritic cells etc. they induce the secretion of type 2 T-helper cells. These activated type 2 T-helper cells influence the production of cytokines like IL-4, IL-5, IL-9 and IL-13. Among these cytokines IL-4, IL-9 and IL-13 binds to B-lymphocytes stimulating the expression of $\operatorname{IgE}$ antibodies. Further, these IgE antibodies bind to high affinity IgE receptor i.e. FceRI on target mast cells. This high affinity IgE receptor activates sphingosine kinase dependent calcium mobilization in mast cells leading to degranulation with the release of inflammatory mediators like histamine, prostaglandin 
D2 and leukotrienes which in turn acts on smooth muscle cells to induce bronchoconstriction. On the other hand, IL-5 and IL- 9 act on eosinophils causing their activation, maturation and differentiation and finally leads to tissue damage [10].

Chronic Obstructive Pulmonary Disease: Cytokines released predominantly from $\mathrm{T}$ cells orchestrate the inflammation. Increased expression of IL-4 occurs in BAL fluid from patients with COPD. IL-4 is crucial for differentiation of Th2 cells from Th0 cells and may be important in initial sensitization to allergens. IFN- $\gamma$ is the predominant cytokine in inflammation in patients and it orchestrates the infiltration of Th1 and Tc cells in the lungs through up-regulation of chemokine receptor CXCR3 on these cells and with the release of CXCR3 activating chemokines like CCL9, CCL10 and CCL11. Increased expression of IL-18 occurs in the alveolar macrophages in the airways of COPD patients and has been linked to disease severity. Sputum and BAL fluid express increased amount of pro-inflammatory cytokines TNF- $\alpha$, IL-1 and IL-6. Elevated levels of chemokines like CCL2 also appear in sputum, BAL fluid and lungs of COPD patients and are also expressed by alveolar macrophages, T cells and epithelial cells [3]

HIV Infection: HIV infection results in dysregulation of the cytokine profile both in vitro and in vivo. An important role is played by cytokines in controlling the homeostasis of the immune system. Secretion of Th1 cytokines such as IL-2 and IFN- $\gamma$ is decreased while production of Th2 cytokines IL-4, IL-10 and proinflammatory cytokine like IL-1, IL-6, IL- 8 and TNF- $\alpha$ is increased at the time of HIV infection. Further, TNF- $\alpha$, TNF- $\beta$, IL-1 and IL- 6 stimulate HIV replication in T cells and monocyte derived macrophages (MDM). So this type of an abnormal cytokine production impairs the cell mediated immunity thus contributing to the pathogenesis of the disease. IL-2, IL-7 and IL-5 up-regulates HIV-1 in T cells while macrophage colony stimulating factor (M-CSF) stimulates HIV in MDM. IFN- $\alpha$, IFN-, and IL-16 are HIV suppressive cytokines which inhibit HIV-1 replication in T cells and MDM whereas, IL-10 and IL-13 inhibits it in MDM only. IFN- $\gamma$, IL-4 and GM-CSF, which are bi-functional cytokines, have shown both inhibitory and stimulatory effects for HIV infection [11].

Rheumatoid arthritis: This is a chronic autoimmune disease presenting with stiffness, pain and symmetrical synovitis of diarthrodial joints. Many cytokines get involved in early events in the rheumatoid synovium. The mechanism of rheumatoid synovitis remains unclear, but both antigen-specific and non-antigen-specific mechanisms may be involved. The pathophysiologic process may be initiated by activated T-cells, expressing HLA-DR4 with the MHC-shared epitope [12]. T-cell antigens which are responsible remain elusive, and they may be multiple, such as viral or bacterial products. The stimulated $\mathrm{T}$ cells may cross-react with self-antigens, resulting in the activation of macrophages by the secretion of cytokines like IFN $-\gamma$ or through direct cell-to-cell contact. The initiation of rheumatoid synovitis by non-antigen-specific mechanisms involves episodic release of TNF- $\alpha$ and GM-CSF from synovial fibroblasts and macrophages, and may be induced by infections, minor trauma, vaccinations, allergic responses, or local immune complex deposition [13]. The dendritic cells are differentiated by these cytokines into potent antigen-presenting cells, which may selectively present self-antigens for the induction of specific T-cell responses. The presence of the shared epitope reduces the transformation of acute reactive synovitis to rapidly destructive synovial reaction by increasing the presentation of self-antigens by the dendritic cells.

Cytokines are involved in the next phase of rheumatoid synovitis that may be characterized by the migration of immune and inflammatory cells from the blood to synovial space and tissue. TNF- $\alpha$ and IL-1, as well as GM-CSF and IL-8, from activated macrophages increase the adhesion molecules expression on endothelial cells in synovial post-capillary venules [14]. Nucleated cells in the blood attach to these stimulated endothelial cells and migrate into the synovium under the influence of chemokines such as IL-8. IL-8 is a major chemokine involved in rheumatoid arthritis, but some other chemokines are also present in the synovial fluid of rheumatoid arthritis patients, like MIP- $1 \alpha$ and MCP-1 [15]. Chemokines in the rheumatoid synovium are mainly derived from macrophages and fibroblasts. These molecules then infiltrate monocytes, T-cells and neutrophils into the rheumatoid synovium, where they are induced by other cytokines.

Cytokines in brain: Various cytokines and their receptors are constitutively expressed and secreted in the normal human brain and their levels can vary in astrocytes and microglia. However, in rodents the maximum densities of IL-1, IL-2, IL-6, and TNF- $\alpha$ receptors was detected in hippocampus and hypothalamus [16]. According to age the levels of various cytokines increases in the CNS. Increased expression of IL-1 and microglial activation was seen with age in neurologically intact patient's brain [17]. Such age-related increased cytokine level may result in increased risk of neurodegenerative responses.

Cytokines, particularly those of the neuropoietic, or gp130 family, regulate the neuroepithelial/radial glia cells and their self-renewal. These cytokines in the brain also function as the scaffolds for migrating neurons and as the precursors for all macroglia, neurons (astrocytes and oligodendrocytes), and adult progenitors. Radial glial cells first give rise to neurons and then glia, and other cytokines, including the gp130. The bone morphogenetic proteins have a major role in the formation of glia from the neuron. Chemokines give cue for the migration of newly formed neurons and glia and are modulators of axon path finding. A recent animal study showed that dysregulation of maternal cytokines may induce striking behavioral deficits in the offspring [1].

In hippocampal neurons, TNF $\alpha$ modulates synaptic strength by promoting surface expression of AMPA-type glutamate receptors (AMPAR) [18]. Cingolani et al. [19] demonstrated that TNF $\alpha$ mediates this effect through a process that involves the upregulation of $\beta 3$ integrin expression on AMPARs.

Multiple sclerosis: Multiple sclerosis is an autoimmune neurodegenerative disease of the white matter of the CNS. Neurodegeneration is a consequence of demyelination which generates the plaques in the white matter and is a characteristic pathology of the disease. IL- 6 and IFN- $\gamma$ trigger an inflammatory response in the white matter of the brain leading to the development of plaques. Administration of IFN- $\gamma$ exacerbates multiple sclerosis. IFN- $\gamma$ is the most popular treatment which 
is mildly successful in reducing the relapse rate in patients with multiple sclerosis. IFN- $\gamma$, the signature cytokine for Th1 cells, is capable of inducing worsening of multiple sclerosis. TGF- $\beta$ may be involved in suppression of inflammation late in the chronic stage of the disease. However, activation of TGF- $\beta 1$ is associated with heightened inflammation in the relapsing remitting early phase of multiple sclerosis. Blockade of TNF- $\alpha$ is again associated with worsening of multiple sclerosis. Recent research has demonstrated that IL-17 is present in multiple sclerosis lesions. In addition, receptors for IL-17 and IL-22 are also present on endothelial cells at the blood brain barrier [20].

Alzheimer disease: Alzheimer disease is the most common form of dementia occurring in the aged population. It is characterized by progressive memory deficits, cognitive impairment and personality changes. Research is still on progress regarding the role of various cytokines in Alzheimer disease. Pro-inflammatory cytokines, notably IL- $1 \alpha$ and IL- $1 \beta$, have been found throughout the brain of individuals with this condition. Animal models revealed that TGF- $\beta$ signaling was involved in the pathogenesis of the disease. It was further shown that inhibition of TGF- $\beta$ signaling was associated with reduced inflammation in the brain in animal models of Alzheimer disease [20].

Fibrosis: Interstitial fibrosis is commonly characterized by the abnormalactivation offibroblastic cells and excessive accumulation of functionally unfavorable collagenous extracellular matrix. Cytokines stimulate the expression of multiple genes involved in extracellular matrix production and deposition of collagen and proteoglycans. They affect the proliferation, migration and differentiation of epithelial cells [21]. Transforming growth factor, which is a ubiquitous cytokine, contributes to fibroblast activation, collagen overproduction and tissue fibrosis [22]. This collagen substrate results in apoptosis of epithelial cells in the presence of TGF- $\beta$. So, TGF- $\beta$ has anti-proliferative effects in most epithelial and endothelial cells which could promote differentiation. Hence TGF- $\beta$ is a classical example of profibrotic growth factor that is regulated by extracellular matrix [21].

Other diseases: IL-23 pathway and Th cells induction and regulation are involved in the pathogenesis of psoriasis vulgaris and psoriatic arthritis. Psoriatic skin is characterized by severe inflammation and hyperproliferative, poorly differentiated keratinocytes. IL-17 and IL-22 can result in hyperproliferative keratinocytes and synoviocytes leading to cellular proliferation and inflammation in both skin and joints. Further, the disease related cytokines in synovial tissue could also promote formation of osteoclast that results in bone erosion [23].

TNF- $\alpha$ in monocytes is independently associated with stroke and stroke associated infection. The number of monocytes is elevated in patients with severe stroke or stroke associated infection. Stroke associated infection may result from the immunosuppressive and anti-inflammatory effects of corticoids, catecholamines, IL-10 and deactivated monocytes [24].

IL-1 $\beta$ expression by HIV infected cells may be one of the important factors for induction of HIV encephalitis. Cytokines such as TNF- $\alpha$ and IL- $1 \beta$ have been thought to have toxic effects on CNS cells and is also involved in the induction of neuronal death. The release of cytokines in HIV encephalitis is mainly mediated by macrophage/microglia lineage. Further, non-infected macrophages/microglia as well as some astrocytes express IL-1 $\beta$ and TNF- $\alpha$ which might have a role in the pathogenesis of AIDS dementia complex (ADC) [25].

Vascular endothelial growth factor (VEGF) plays a critical role in inflammatory response in atherosclerosis, sepsis and rheumatoid arthritis. VEGF via VEGF receptor2 predominantly induces the production of pro-inflammatory cytokines IL-6, IL-8 and growth related oncogene-alpha in endothelial cells. So, this explains a new insight into the mechanism of the proinflammatory activity of VEGF in host inflammatory responses in several disease states [26].

Arsenicosis is a multisystem disorder that has been linked to hyperkeratosis and skin cancer. Cutaneous toxicity is a related effect of various cytokines like IL-8, TGF- $\beta$, TNF- $\alpha$ and GM-CSF. Arsenic causes apoptosis via generation of free radicals. The histopathological findings of hyperkeratosis and dysplastic cells in the arsenicosis skin lesions can be related to increased expression of cytokeratins, keratin-16 which is a marker for hyperproliferation and keratin-8 and -18 which are markers for less differentiated epithelial cells [27].

Psychological stress is a potent inducer of anti-inflammatory cytokine IL-10 and it homolog IL-19 via activation of $\beta$-adrenoceptors, and may be key mediators of stress-induced immunosuppression. Research findings suggest that stress enhances the production of immunosuppressive cytokines which might have an impact on stress-related disease processes [28].

\section{Cytokines as therapeutic targets}

IL-1 and IL-2 showed potential as natural immunostimulants to combat the immune deficiency of AIDS. Clinical and experimental studies supported the hypothesis that immunestimulant cytokines could be helpful in neutralizing the immunesuppression of cancer and AIDS.

Similar effects were also proposed for TNF- $\alpha$ for cancer patients. However, the disturbing inflammatory response of cytokines like IL-1, IL-2, IL-3, IL-4, IL-6, IL-12 or TNF- $\alpha$ in human resulted in the side lining of such therapy. The only cytokine to receive approval for treating cancer is IL-2 but its pro-inflammatory effects were not well tolerated and hence their efficacy in conditions like melanoma and renal cell carcinoma was low. IL-10 was a good candidate for a variety of autoimmune diseases, since it suppressed IFN $\gamma$, IL-1, TNF- $\alpha$, and IL- 6 production as well as possessing other anti-inflammatory activities. Several trials of recombinant human IL-10 showed limited efficacy in psoriasis, rheumatoid arthritis and Crohn's disease but the cytokine, was never been approved for therapeutic use. On the other hand, colony stimulating factors such as G-CSF or GM-CSF are used to treat bone marrow suppression associated with radiation, chemotherapy or transplantation. GM-CSF has also been used to treat Crohn's Disease. Erythropoietin (EPO) is routinely used to large numbers of patients with anemia and bone marrow failure. Further, IFN $\alpha$ is administered to patients of hepatitis B and C. IFN $\beta$ for the treatment of multiple sclerosis is also effective [5]. 
As it is confirmed from previous evidence that cytokines are involved in every important biological process, cytokine antagonist therapy is a rapidly growing and advancing field in clinical practice with great pharmaceutical impact. Because cytokines are potential rate-limiting molecules in various diseases, therefore, they are the excellent targets for the biotechnology products like monoclonal antibodies and antibody-like receptor: Fc fusion proteins for the pharmaceutical industry [29]. One of the very initial applications showed that treatment with TNF- $\alpha$ blocker suppressed disease activity and attenuated joint deformation and destruction in rheumatoid arthritis- highlighting the importance of cytokine antagonists in disease [30,31].

Treatment with Afelimomab, an anti-TNF- $\alpha$ antibody in patients of severe sepsis showed significant attenuation in IL-6, TNF- $\alpha$ levels and severity of organ dysfunction [32]. Treatment with etanercept, a TNF- $\alpha$ antagonist, for twelve weeks in patients of refractory asthma showed significant improvement in asthma control and systemic inflammation [33]. IL-6 blockade showed beneficial effects in both experimental models and in human disease and inhibition of IL-6 signaling with tocilizumab could reverse or prevent symptoms typically associated with rheumatic diseases [34]. Trastuzumab (Herceptin), which is HER2-specific monoclonal antibody, is used for the treatment of adenocarcinoma of the stomach or the gastroesophageal junction and also aggressive form of breast cancer $[35,36]$.

G-CSF is used clinically in idiopathic neutropenia, congenital neutropenia, febrile neutropenia, leukemic neutropenia, and aplastic anemia to increase the PMNL count, which improves the quality of life and reduce the morbidity and mortality in affected individuals [37].

Table 1: Cytokines as targets for therapeutic treatment of diseases.
FDA approved the use of IFN- $\gamma$ for the treatment of chronic granulomatous disease (CGD) [38]. IFN- $\gamma$ also has an immunomodulatory effect in other specific immunodeficiency syndromes. It is an effective adjunctive therapeutic agent in combination with other conventional antimicrobial agents in cutaneous and visceral leishmaniasis, disseminated atypical mycobacterial infection, or lepromatous leprosy patients [3941]. It also activates alveolar macrophages and plays an effective role in host immunity against M. tuberculosis. IFN- $\gamma$ as an aerosol form has been used in multidrug-resistant tuberculosis patients [42]. Antibodies against cytokines like IL-4, IL-5, IL-13 are used for the treatment of asthma. IL-10, IL-11, anti- IL-12, anti-TNF- $\alpha$ are used for the prevention of psoriasis. Cytokines which are antiinflammatory in nature, like IL-10 and IL-11, are used in Crohn's disease and ulcerative colitis [2]. Treatment with altrakincept, a recombinant human soluble IL-4 receptor, neutralizes endogenously produced IL-4 in bronchial asthma patients [43]. Treatment with monoclonal antibody against IL-5 showed reduction in the numbers of blood and sputum eosinophils, airway hyperresponsiveness and late asthmatic response in asthmatic patients [44]. In various animal and in-vitro studies, administration of anti-IL-13 antibody prevents or reverses the symptoms of bronchial asthma [45].

Cytokines IL-2 and IFN- $\alpha$ are approved by FDA, IL-2 for the metastatic melanoma and renal cell carcinoma treatment in highdose bolus and IFN- $\alpha$ as an adjuvant therapy of Stage III melanoma, hematologic malignancies, AIDS-related Kaposi's sarcoma, and with bevacizumab as an anti-angiogenic for advanced renal cancer. Recently various cytokines viz. IL-7, IL-12, IL-15, IL-18, IL21 and GM-CSF, are under clinical trials for advanced cancer [46] (Table 1).

\begin{tabular}{|c|c|}
\hline Cytokines & Disease Targets \\
\hline IFN- $\alpha$ & Hepatitis B and C [5] \\
\hline IFN- $\beta$ & Multiple sclerosis [5] \\
\hline IFN- $\gamma$ & Chronic granulomatous disease [38], Crohn's disease [65], Multidrug resistant tuberculosis [42] \\
\hline TNF- $\alpha$ & Rheumatoid arthritis [30,31], Sepsis [32], Refractory asthma [33], Psoriasis [2] \\
\hline G-CSF & $\begin{array}{l}\text { Idiopathic neutropenia, Congenital neutropenia, Febrile neutropenia, Leukemic neutropenia, Aplastic } \\
\text { neutropenia [37], Bone marrow suppression [5] }\end{array}$ \\
\hline GM-CSF & Bone marrow suppression, Crohn's disease [5] \\
\hline IL-2 & Metastatic melanoma, Renal cell carcinoma [46] \\
\hline IL-4 & Asthma [43] \\
\hline IL-5 & Asthma [44] \\
\hline IL-6 & Rheumatic diseases [34] \\
\hline IL-10 & Crohn's disease, Ulcerative colitis [2], Psoriasis, Rheumatoid arthritis [5] \\
\hline IL-11 & Crohn's disease, Ulcerative colitis, Psoriasis, Rheumatoid arthritis [2] \\
\hline IL-13 & Asthma $[2,45]$ \\
\hline Erythropoietin & Anaemia, Bone marrow failure [5] \\
\hline $\begin{array}{l}\text { HER2-Specific Monoclonal } \\
\text { Antibody }\end{array}$ & Adenocarcinoma of stomach, Breast cancer $[35,36]$ \\
\hline
\end{tabular}




\section{Toxicity during cytokine therapy}

Therapy with cytokines often leads to dose-dependent side effects. Being pleiotropic in nature, cytokines are able to influence more than a single cell. Because of their short half-life, for better therapeutic effects it requires high doses, which may cause pleiotropic activities and ultimately lead to adverse effects [47]. Administration of high dose of IL-2 for cancer immunotherapy stimulates the cytotoxic CD8+ T-cells and NK cells proliferation, which promotes tumor regression in these patients and leads to adverse reactions [48].

Increased IL-8 or TNF- $\alpha$ in maternal serum is associated with an increased risk for schizophrenia in the offspring [48-51]. Common side effects with G-CSF, shown by $20-30 \%$ of patients, are mild-to-moderate bone and musculoskeletal pain [51,52]. In addition to these effects, there are relatively few adverse effects, even after years of administration in patients with severe chronic neutropenia [53]. Other side effects due to G-CSF are headache, splenomegaly, anemia, thrombocytopenia, hypersensitivity and urticarial at injection site [52,54]. G-CSF-also induced lung toxicity post cancer chemotherapy [55-57].

GM-CSF treatment has greater frequency of side effects -most common being fever accompanied with influenza-like syndrome and myalgias [58]. Flushing, hypotension, tachycardia, dyspnea and musculoskeletal pain are the first dose reaction due to the GM-CSF administration. Arterial oxygen desaturation, nausea and vomiting occurred in 5\% patients, and at high dose generalized capillary leak syndrome may occur $[59,60]$.

IFN- $\gamma$ administration may cause hypersensitivity reaction, chills, fatigue, fever, myalgias, and headache associated with mild influenza-like symptoms and can be managed with prophylactic antipyretics $[38,39]$. However, administration through inhalation for the treatment of pulmonary infections, is associated with lesser side effects [42]. IFN- $\gamma$ treatment is effective in rheumatoid arthritis and systemic sclerosis patients, but it can also exacerbate multiple sclerosis [39]. Hence, it should be used with caution in such patients or in patients with inflammatory disorder.

IFN- $\alpha$ induced toxicity is usually dose-related, and can be managed without discontinuation of treatment. The common side effects are headaches, fever, fatigue, myalgias and abdominal disturbance which occur in more than $80 \%$ of patients. Few patients show elevated hepatic enzymes during the high-dose intravenous administration, and thus such patients should be monitored for liver function. Leukopenia, neutropenia and thrombocytopenia are other common side effects that can be readily managed via dose reduction. Central symptoms include mania in lower than $1 \%$, confusion in $10 \%$, and depression in $45 \%$ of patients during therapy [61-63]. Alterations of the immune system, which can be persisting even after discontinuation of the therapy, are also seen in few cases. Vitiligo, hypothyroidism, occurrence of sarcoidosis, lupus, rheumatoid arthritis and psoriasis are also observed in these patient [64].

\section{Conclusion}

In conclusion, cytokines are ubiquitous molecules which act as key messengers for and between immune cells and help to maintain a delicate and intricate balance in the immune system. Cytokines affect nearly every biological process viz. embryonic development, disease pathogenesis, non-specific response to infection, specific response to antigen, changes in cognitive functions and progression of the degenerative processes of aging. They are also involved in stem cell differentiation, vaccine efficacy and allograft rejection. Such regulation of immune homeostasis is crucial for health and disease, and disruption of this balance results in many chronic pathophysiological states. Thus, it is imperative that therapeutic targeting of cytokine pathways holds great promises for patients suffering from several intractable chronic diseases.

\section{References}

1. Deverman BE, Patterson PH (2009) Cytokines and CNS Development. Neuron 64(1): 61-78.

2. Zídek Z, Anzenbacher P, Kmonickoval E (2009) Current status and challenges of cytokine pharmacology. Br J Pharmacol 157(3): 342361.

3. Barnes PJ (2008) The cytokine network in asthma and chronic obstructive pulmonary disease. J Clin Invest 118(11): 3546-3556.

4. Owen JA, Punt J, Stranford SA (2013) Kuby Immunology. WH Freeman \& Company, New York, USA.

5. Dinarello CA (2007) Historical Review of Cytokines. Eur J Immunol 37(Suppl 1): S34-S45.

6. Wilson CJ, Finch CE, Cohen HJ (2002) Cytokines and cognition-the case for a head-to-toe inflammatory paradigm. J Am Geriatr Soc 50(12): 2041-2056.

7. Kips JC (2001) Cytokines in asthma. Eur Respir J 18(34): 24s-33s.

8. Steinke JW, Borish L (2001) Th2 cytokines and asthma Interleukin-4: its role in the pathogenesis of asthma, and targeting it for asthma treatment with interleukin-4 receptor antagonists. Respir Res 2(2): 66-70.

9. Chung F (2001) Antiinflammatory cytokines in asthma and allergy: interleukin-10, interleukin-12, interferon $\gamma$. Mediators Inflamm 10(2): 51-59.

10. Mahajan S, Mehta AA (2006) Role of cytokines in pathophysiology of asthma. Iran J Pharmacol Therap 5: 1-14.

11. Kedzierska K, Crowe SM (2001) Cytokines and HIV-1: Interactions and clinical implications. Antivir Chem Chemother 12(3): 133-150.

12. Fox DA (1997) The role of $T$ cells in the immunopathogenesis of rheumatoid arthritis: new perspectives. Arthritis Rheum 40(4): 598-609.

13. Thomas R, Lipsky PE (1997) Could endogenous self-peptides presented by dendritic cells initiate rheumatoid arthritis? Immunol Today 17(12): 559-564.

14. Mojcik CF, Shevach EM (1997) Adhesion molecules: a rheumatologic perspective. Arthritis Rheum 40(6): 991-1004.

15. Kunkel SL, Lukacs N, Kasama T, Streiter RM (1996) The role of chemokines in inflammatory joint disease. J Leukoc Biol 59(1): 6-12.

16. Vitkovic L, Boackaert J, Jacque C (2000) Inflammatory cytokines: neuromodulators in normal brain? J Neurochem 74(2): 457-471.

17. Sheng JG, Mrak RE, Griffin WS (1998) Enlarged and phagocytic, but not primed, interleukin-1 alpha-immunoreactive microglia increase with age in normal human brain. Acta Neuropathol 95(3): 229-234.

18. Beattie EC, Stellwagen D, Morishita W, Bresnahan JC, Ha BK, et al. 
(2002) Control of synaptic strength by glial TNF alpha. Science 295(5563): 2282-2285.

19. Cingolani LA, Thalhammer A, Yu LM, Catalano M, Ramos T, et al. (2008) Activity-dependent regulation of synaptic AMPA receptor composition and abundance by beta3 integrins. Neuron 58(5): 749762.

20. Steinman L (2008) Nuanced role of cytokines in three major human brain disorders. J Clin Invest 118(11): 3557-3563.

21. Sivakumar P, Das AM (2008) Fibrosis, chronic inflammation and new pathways for drug discovery. Inflamm Res 57(9): 410-418.

22. Varga J (2008) Systemic sclerosis: an update. Bull NYU Hosp Jt Dis 66(3): 198-202.

23. Nograles KE, Brasington RD, Bowcock AM (2009) New insights into the pathogenesis and genetics of psoriatic arthritis. Nat Clin Pract Rheumatol 5(2): 83-91.

24. Urra X, Cervera A, Obach V, Climent N, Planas AM, Chamorro A (2009) Monocytes are major players in the prognosis and risk of infection after acute stroke. Stroke 40(4): 1262-1268.

25. Xing HQ Hayakawa H, Izumo K, Kubota R, Gelpi E, et al. (2009) In vivo expression of proinflammatory cytokines in HIV encephalitis: an analysis of 11 autopsy cases. Neuropathology 29(4): 433-442.

26. Hao Q, Wang L, Tang H (2009) Vascular endothelial growth factor induces protein kinase D dependent production of proinflammatory cytokines in endothelial cells. Am J Physiol Cell Physiol 296(4): 821-827.

27. Sengupta SR, Das NK, Datta PK (2008) Pathogenesis, clinical features and pathology of chronic arsenicosis. Indian J Dermatol Venereol Leprol 74(6): 559-570.

28. Curtin NM, Mills KH, Connor TJ (2009) Psychological stress increases expression of IL-10 and its homolog IL-19 via beta adrenoceptor activation: Reversal by the anxiolytic chlordiazepoxide. Brain Behav Immun 23(3): 371-379.

29. Feldmann M (2008) Many cytokines are very useful therapeutic targets in disease. J Clin Invest 118(11): 3533-3536.

30. Elliott MJ, Maini RN, Feldmann M, Lonq-Fox A, Charles P, et al. (1993) Treatment of rheumatoid arthritis with chimeric monoclonal antibodies to tumor necrosis factor alpha. Arthritis Rheum 36(12): 1681-1690.

31. Feldmann M, Maini RN (2001) Anti-TNF alpha therapy of rheumatoid arthritis: what have we learned. Annu Rev Immunol 19: 163-196.

32. Panacek EA, Marshall JC, Albertson TE, Johnson DH, Johnson S, et al. (2004) Efficacy and safety of the monoclonal anti-tumor necrosis factor antibody $\mathrm{F}\left(\mathrm{ab}^{\prime}\right) 2$ fragment afelimomab in patients with severe sepsis and elevated interleukin-6 levels. Crit Care Med 32(11): 2173-2182.

33. Morjaria JB, Chauhan AJ, Babu KS, Polosa R, Davies DE, et al. (2008) The role of a soluble TNFa receptor fusion protein (etanercept) in corticosteroid refractory asthma: a double blind, randomised, placebo controlled trial. Thorax 63(7): 584-591.

34. Lipsky PE (2006) Interleukin-6 and rheumatic diseases. Arthritis Res Ther (Suppl 2): S4.

35. Hudis CA (2007) Trastuzumab- Mechanism of Action and Use in Clinical Practice. N Engl J Med 357(1): 39-51.

36. Hudziak, RM, Lewis GD, Winget M, Fendly BM, Shepard HM, et al. (1989) p185HER2 monoclonal antibody has antiproliferative effects in vitro and sensitizes human breast tumor cells to tumor necrosis factor. Mol Cell Biol 9(3): 1165-1172.

37. Hubel K, Dale DC, Liles WC (2002) Therapeutic Use of Cytokines to Modulate Phagocyte Function for the Treatment of Infectious Diseases: Current Status of Granulocyte Colony-Stimulating Factor, Granulocyte-Macrophage Colony-Stimulating Factor, Macrophage Colony-Stimulating Factor, and Interferon- $\gamma$. J Infect Dis 185(10): 1490-1501.

38. Gallin JL, Farber JM, Holland SM, Nutman TB (1995) Interferongamma in the management of infectious diseases. Ann Intern Med 123(3): 216-224.

39. Murray HW (1994) Interferon-gamma and host antimicrobial defense: current and future clinical applications. Am J Med 97(5): 459-467.

40. Holland SM, Eisenstein E, Kuhns DB, Turner ML, Fleisher TA, et al. (1994) Treatment of refractory disseminated nontuberculous mycobacterial infection with interferon gamma. $\mathrm{N}$ Engl J Med 330(19): 1348-55.

41. Badaro R, Falcoff E, Badaro FS, Carvalho EM, Pedral-Sampaio D, et al. (1990) Treatment of visceral leishmaniasis with pentavalent antimony and interferon-gamma. N Engl J Med 322(1): 16-21.

42. Condos R, Rom WN, Schluger NW (1997) Treatment of multidrugresistant pulmonary tuberculosis with interferon-gamma via aerosol. Lancet 349(9064): 1513-1515.

43. Hendeles L, Asmus M, Chesrown S (2004) Evaluation of cytokine modulators for asthma. Paediatr Respir Rev 5 (Suppl A): S107-S112.

44. Leckie MJ, ten Brinke A, Khan J, Diamant Z, OConnor BJ, et al. (2000) Effects of an interleukin-5 blocking monoclonal antibody on eosinophils, airway hyperresponsiveness, and the late asthmatic response. Lancet 356(9248): 2144-2148.

45. Vugmeyster Y, Szklut P, Tchistiakova L, Abraham W, Kasaian M, et al. (2008) Preclinical pharmacokinetics, interspecies scaling, and tissue distribution of humanized monoclonal anti-IL-13 antibodies with different IL-13 neutralization mechanisms. Int Immunopharmacol 8(3): 477-483.

46. Lee S, Margolin K (2011) Cytokines in cancer immunotherapy. Cancers 3(4): 3856-3893.

47. Kontermann RE (2011) Strategies for extended serum half-life of protein therapeutics. Curr Opin Biotechnol 22(6): 868-876.

48. Boyman O, Surh CD, Sprent J (2006) Potential use of IL-2/antiIL-2 antibody immune complexes for the treatment of cancer and autoimmune disease. Expert Opin Biol Ther 6(12): 1323-1331.

49. Brown AS, Hooton J, Schaefer CA, Zhang H, Petkova E, et al. (2004) Elevated maternal interleukin-8 levels and risk of schizophrenia in adult offspring. Am J Psychiatry 161(5): 889-895.

50. Buka SL, Tsuang MT, Torrey EF, Klebanoff MA, Wagner RL, et al. (2001) Maternal cytokine levels during pregnancy and adult psychosis. Brain Behav Immun 15(4): 411-420.

51. Anderlini P, Przepiorka D, Seong D, Miller P, Sundberg J, et al. (1996) Clinical toxicity and laboratory effects of granulocytecolony-stimulating factor (filgrastim) mobilization and blood stem cell apheresis from normal donors, and analysis of charges for the procedures. Transfusion 36(7): 590-595.

52. Price TH, Bowden RA, Boeckh M, Bux J, Nelson K, et al. (2000) Phase 
I/II trial of neutrophil transfusions from donors stimulated with G-CSF and dexamethasone for treatment of patients with infections in hematopoietic stem cell transplantation. Blood 95(11): 33023309.

53. Freedman MH (1997) Safety and long-term administration of granulocyte colony- stimulating factor for severe chronic neutropenia. Curr Opin Hematol 4(3): 217-224.

54. Stroncek DF, Yau YY, Oblitas J, Leitman SF (2001) Administration of G-CSF plus dexamethasone produces greater granulocyte concentrate yields while causing no more donor toxicity than G-CSF alone. Transfusion 41(8): 1037-1044.

55. Katoh M, Takada M, Nakayama M, Umeda M (1996) Pulmonary toxicity during granulocyte colony-stimulating factor administration and neutrophils. Chest 110: 576-587.

56. Niitsu N, Iki S, Muroi K, Motomura S, Murakami M, et al. (1997) Interstitial pneumonia in patients receiving granulocyte colonystimulating factor during chemotherapy: survey in Japan 1991-96. Br J Cancer 76(12): 1661-1666.

57. Hilbe W, Nussbaumer W, Bonatti H, Thaler J, Niederwieser D, et al. (2000) Unusual adverse events following peripheral blood stem cell (PBSC) mobilisation using granulocyte colony stimulating factor (GCSF) in healthy donors. Bone Marrow Transplant 26: 811-823.

58. Peters WP, Shogan JS, Shpall EJ, Jones RB, Kim CS (1988) Recombinant human granulocyte-macrophage colony-stimulating factor produces fever. Lancet 1(8591): 950.
59. Lieschke GJ, Cebon J, Morstyn G (1989) Characterization of the clinical effects after the first dose of bacterially synthesized recombinant granulocyte macrophage colony-stimulating factor. Blood 74: 2634-2643.

60. Arnig M, Kliche KO, Schneider W (1991) GM-CSF therapy and capillary-leak syndrome. Ann Hematol 62(2-3): 83.

61. Jonasch E, Haluska FG (2001) Interferon in oncological practice: Review of interferon biology, clinical applications, and toxicities. Oncologist 6(1): 34-55.

62. Jonasch E, Kumar UN, Linette GP, Hodi FS, Soiffer RJ, et al. (2000) Adjuvant high-dose interferon alfa- $2 \mathrm{~b}$ in patients with high-risk melanoma. Cancer J 6(3): 139-145.

63. Greenberg DB, Jonasch E, Gadd MA, Ryan BF, Everett JR, et al (2000) Adjuvant therapy of melanoma with interferon-alpha-2b is associated with mania and bipolar syndromes. Cancer 89(2): 356362.

64. Jones TH, Wadler S, Hupart KH (1998) Endocrine-mediated mechanisms of fatigue during treatment with interferon-alpha. Semin Oncol 25 (1 Suppl 1): 54-63.

65. Hommes DW, Mikhajlova TL, Stoinov S, Stimac D, Vucelic B, et al. (2006) Fontolizumab, a humanised anti-interferon gamma antibody, demonstrates safety and clinical activity in patients with moderate to severe Crohn's disease. Gut 55(8): 1131-1137. 